\title{
Geometric effects in continuous-media percolation
}

\author{
Ping Sheng and R. V. Kohn* \\ Corporate Research Science Laboratories, Exxon Research and Engineering Co., \\ P. O. Box 45, Linden, New Jersey 07036
}

(Received 11 February 1982)

\begin{abstract}
We study the simple system of a two-dimensional square lattice composed of goodconductor and poor-conductor squares, with the use of a clustered mean-field approximation. Instead of the well-known threshold behavior predicted by the two-component site percolation model or the effective-medium theory, we find two conductivity percolation thresholds at which the real and imaginary parts of the effective dielectric constant exhibit distinct critical behaviors. The cause of this double-threshold characteristic is shown to be the existence of a third conductivity scale arising from the corner-corner interactions between second-nearest-neighbor squares. Analogies with site percolation models are also detailed. It is demonstrated that as $\left|\epsilon_{1} / \epsilon_{2}\right| \rightarrow \infty$, where $\epsilon_{1(2)}$ is the complex dielectric constant of the good (poor) conductor, the continuum system can be made equivalent to two versions of the square-lattice site percolation model, depending on whether $\left|\epsilon_{1}\right| \rightarrow \infty$ or $\left|\epsilon_{2}\right| \rightarrow 0$. The paper concludes with a discussion of possible implications for three-dimensional continuous-media percolating systems.
\end{abstract}

Percolation is a subject common to a wide variety of physical problems involving the macroscopic properties of inhomogeneous media. In the past decade, extensive theoretical study on discrete percolating networks consisting of nodes and bonds has elucidated many fundamental characteristics of these systems. ${ }^{1}$ However, it has been pointed out ${ }^{2}$ that in real composite materials with space- (area-) filling constituents, the percolation properties can be drastically different from the predictions of the network models. The discrepancies occur in part because the components of a continuous-media percolating system can have various geometric shapes and correlated arrangements, ${ }^{3}$ features that are difficult to model by any simple discrete network. The work reported herein indicates that such geometric effects may not only shift the value of the percolation threshold, but may also induce critical behavior at more than one threshold for a two-component composite. In fact, our simple model displays a distinct type of critical behavior for the real and imaginary parts of the dielectric constant at each of the two thresholds.

We consider a two-dimensional simple square lattice in which each square has the probability $p$ of being occupied by material 1 and probability $1-p$ of being occupied by material 2 . Since we are interested in the electrical properties of the system, each material is characterized by a complex dielectric constant $\epsilon_{1(2)}=\delta_{1(2)}+i\left(4 \pi \sigma_{1(2)} / \omega\right)$, where $\delta_{1(2)}$ is the real part of the dielectric constant for ma- terial $1(2), \sigma_{1(2)}$ is the conductivity for material 1 (2), and $\omega$ is the angular frequency of the electromagnetic field. To inject an element of realism, we will further characterize the corners of the squares as not being perfectly sharp, but can have a small contact of width $w$ in units where the side of each square is defined as 1 . We are interested in the properties of the system as $w \rightarrow 0$. There are two simple methods in the literature that one can employ to estimate the effective dielectric constant $\bar{\epsilon}$ of this model: the site percolation model, in which the two nearest-neighbor nodes are connected by one of the two types of bonds, and the effective medium theory. ${ }^{2}$ If we assume $\left|\epsilon_{2}\right|<<\left|\epsilon_{1}\right|$, both theories predict that $\ln |\bar{\epsilon}|$ would experience one single sharp variation at the threshold value of $p$. It is the purpose of this paper to point out that the actual behavior of this simple system differs significantly from such prediction. In fact, our calculation demonstrates that due to the cornercorner interaction between the second-nearestneighbor squares, $\ln |\bar{\epsilon}|$ exhibits two sharp variations of equal magnitude: one at $p>0.5$ and the other one located symmetrically below $p=0.5$. To obtain a qualitative understanding of this behavior, we note that a simple square lattice can have two types of connected networks ${ }^{4}$ : the nearest-neighbor network and the second-nearest-neighbor network. It is a matter of simple logic to deduce that the two sharp variations in $\ln |\bar{\epsilon}|$ must be related to the formation of these two types of networks. 
However, what might seem surprising is that the formation of the second-nearest-neighbor conductor network, which is connected only by pointlike contacts (between the conductor square corners), can actually contribute to an increase in $\ln |\bar{\epsilon}|$ that is equal in magnitude to the increase at the formation of the nearest-neighbor conductor network. For example, if $w \rightarrow 0$, we would intuitively expect a negligible amount of current to pass through the corner contacts, and therefore no significant increase in the effective conductivity should exist. But some additional thought would indicate that besides the current which directly passes through the contacts, there should also be a component of the current that goes around the corner contact, through the poor conductor. Solution of the electrostatic problem shows that due to the strong electric field and the short current path near a corner contact, this second component of the current (around the contact) can actually contribute to a "corner conductance" that is equal in magnitude to the geometric mean of $\left|\epsilon_{1}\right|$ and $\left|\epsilon_{2}\right|$ (times the length for the side of a square, which is 1$)$. Therefore, unless $\left|\epsilon_{1}\right| w \geq \sqrt{\left|\epsilon_{1} \epsilon_{2}\right|}$ (or $w \geq \sqrt{\left|\epsilon_{2} / \epsilon_{1}\right|}$ ), the width of the corner contact is immaterial, since the dominant amount of current does not directly pass through the contact anyway. The existence of the corner conductance even in the limit of $w=0$ also implies that if one insists upon using a network percolation model to calculate the behavior of the present system, then a third type of bond, with a conductance value that is the geometric mean of the other two, must be included as a part of the model to account for the second-nearestneighbor interactions (it should be noted, however, that the third conductance is not an independent element since its value is completely determined by the other two). In fact, this third conductance scale is what makes it imperative for the system to have a second threshold.

This paper presents a "clustered-mean-field" calculation of the effecitve dielectric constant for the square lattice. The key element of the schemes is the embedding of corner-corner interaction in a mean field, and imposing a condition of selfconsistency. In what ensues, the consideration of the general formalism is followed by the calculation of $\bar{\epsilon}$ and the discussion of results. The paper concludes with a deliberation on possible implications for three-dimensional percolating systems.

Consider a $2 \times 2$ block of squares in the lattice. In Fig. 1 we enumerate the seven distinct configurations together with their probabilities of oc-
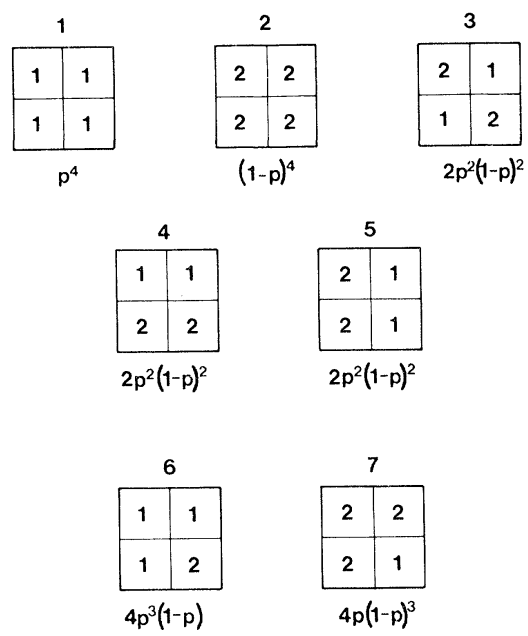

FIG. 1. Seven distinct cluster configurations together with their probabilities of occurrence. The direction of the electric field is assumed to be from left to right.

currence $W_{n}$ (degeneracies included). For the ease of computation, in the following the square geometry of the cluster will be approximated by a quartered circle. The effective dielectric constant is determined by requiring that the mean dipole moment of the seven configurations, when embedded in a homogeneous effective medium, should vanish. To obtain the dipole moment $D_{n}$ of each cluster in a medium of dielectric constant $\bar{\epsilon}$, we have solved the electrostatic problem of a quartered circle of radius 1 in a unit electric field as shown in Fig. 2. The dielectric constant in the ith quadrant is denoted by $\kappa_{i}$, and the exterior dielectric constant is denoted by $\bar{\kappa}$. Since the potential $u$ satisfies the Laplace equation, outside the circle it must have the form

$$
u^{\text {out }}=r \cos \theta+\sum_{n=1}^{\infty}\left(a_{n} \cos n \theta+b_{n} \sin n \theta\right) r^{-n} .
$$

where $r$ is the radial coordinate, $\theta$ the angular coordinate, and $a_{n}, b_{n}$ the expansion coefficients.

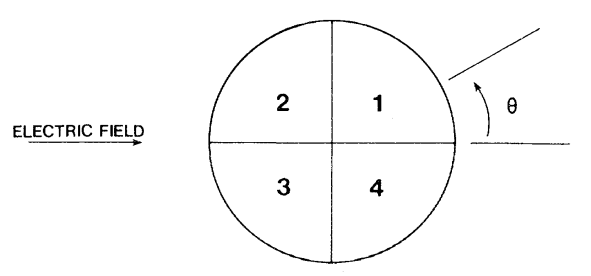

FIG. 2. Quartered circle in a uniform field. 
Inside, we sought a basis of the form $u_{j}=r^{t}\left[A_{j} \exp (i t \theta)+B_{j} \exp (-i t \theta)\right]$ in quadrant $j$, where $A_{j}$ and $B_{j}$ are constants for any given configuration of $\kappa_{j}$. By requiring the potential $u_{j}$ and $\left(\kappa_{j} \partial u_{j} / \partial \theta\right)$ to be continuous across all four dielectic boundaries, one obtains a fourth-order eigenvalue equation for $t$ whose solutions are given by $t=n \pm\left(1-t_{0}\right) \sin (n \pi / 2)$, where $n=1,2,3, \ldots$, and

$$
t_{0}=\frac{1}{\pi} \arccos \left[-\left[1+2 \frac{2-\left(\rho_{1} \rho_{3}+\rho_{2} \rho_{4}\right)}{\left(1+\rho_{1}\right)\left(1+\rho_{2}\right)\left(1+\rho_{3}\right)\left(1+\rho_{4}\right)}\right]\right] \text {. }
$$

Here $\rho_{j}=\kappa_{j} / \kappa_{j+1}\left(\rho_{4}=\kappa_{4} / \kappa_{1}\right)$. If $t$ satisfies Eq. (2), the constants $\left[A_{j}, B_{j}\right]$ may be determined explicitly from $\left[\kappa_{i}\right]$ by solving a set of algebraic equations. Denoting by $u_{l}$ the basis obtained this way, the interior potential can be expanded as

$$
u^{\text {in }}=c_{0}+\sum_{l} c_{l} u_{l}
$$

By matching $u^{\text {in }}$ and $u^{\text {out }}$ through the dielectric boundary conditions at $r=1$, one obtains a set of linear simultaneous equations for the determination of $a_{j}, b_{j}$, and $c_{j}$. Of these coefficients, $a_{1}$ is the dipole moment $D=D\left(\kappa_{1}, \kappa_{2}, \kappa_{3}, \kappa_{4} ; \bar{\kappa}\right)$ of the configuration. One can prove analytically that $D$ has the following symmetries:

$$
D\left(\kappa_{1}, \kappa_{2}, \kappa_{3}, \kappa_{4} ; \bar{\kappa}\right)=D\left(\kappa_{1} / \bar{\kappa}, \kappa_{2} / \bar{\kappa}, \kappa_{3} / \bar{\kappa}, \kappa_{4} / \bar{\kappa} ; 1\right)
$$

and

$$
D\left(\kappa_{1}, \kappa_{2}, \kappa_{3}, \kappa_{4} ; 1\right)=-D\left(\kappa_{2}^{-1}, \kappa_{3}^{-1}, \kappa_{4}^{-1}, \kappa_{1}^{-1} ; 1\right) .
$$

For each of the seven configurations in Fig. 1, the above procedure defines a dipole moment $D_{m}(\bar{\epsilon})$. The condition for determining $\bar{\epsilon}$ is then ${ }^{5}$

$$
\sum_{n=1}^{7} W_{n} D_{n}(\bar{\epsilon})=0
$$

Using the symmetries of $D$, one verifies that this model obeys Keller's theorem ${ }^{6}: \bar{\epsilon}(p) \bar{\epsilon}(1-p)=\epsilon_{1} \epsilon_{2}$, which is known to hold for our continuum problem.

Three of the functions $D_{m}$ are known analytically: $D_{1}=\left(\bar{\epsilon}-\epsilon_{1}\right) /\left(\bar{\epsilon}+\epsilon_{1}\right), D_{2}=\left(\bar{\epsilon}-\epsilon_{2}\right) /\left(\bar{\epsilon}+\epsilon_{2}\right)$ trivially, and from the symmetries of $D$ it can be shown that $D_{3}=\left(\bar{\epsilon}-\sqrt{\epsilon_{1} \epsilon_{2}}\right) /\left(\bar{\epsilon}+\sqrt{\epsilon_{1} \epsilon_{2}}\right)$. We have truncated $u^{\text {in }}$ and $u^{\text {out }}$ to finite number of terms ( 40) and numerically solved Eq. (4). However, when $\left|\epsilon_{2} / \epsilon_{1}\right| \rightarrow 0$, a case of great interest, the numerical solution of Eq. (4) requires solving a poorly conditioned matrix equation. To obtain greater precision and insight, we desired instead a formal asymptotic expansion for $D_{4-7}$ of the form $D_{m} \simeq a+b\left(\bar{\epsilon} / \epsilon_{1}\right)$, neglecting terms of order $\left|\bar{\epsilon} / \epsilon_{1}\right|^{2},\left|\epsilon_{2} / \bar{\epsilon}\right|$, or higher. Expanding the basic elements $u_{l}$ this way, then matching the boundary conditions at $r=1$ term by term in the formal expansion, we found $D_{4}=-0.5+2.36\left(\bar{\epsilon} / \epsilon_{1}\right)$, $D_{5}=0.5+0.64\left(\bar{\epsilon} / \epsilon_{1}\right), D_{6}=-0.71+2.49\left(\bar{\epsilon} / \epsilon_{1}\right)$, and $D_{7}=0.71+0.44\left(\bar{\epsilon} / \epsilon_{1}\right)$. For $p>0.5$, Eq. (4) can now be replaced by an asymptotic equation

$A\left(\frac{\bar{\epsilon}}{\epsilon_{1}}\right)+B+C \frac{\bar{\epsilon}-\sqrt{\epsilon_{1} \epsilon_{2}}}{\bar{\epsilon}+\sqrt{\epsilon_{1} \epsilon_{2}}}+D \frac{\bar{\epsilon}-\epsilon_{1}}{\bar{\epsilon}+\epsilon_{1}}=0$,

with

$$
\begin{aligned}
A= & 6 p^{2}(1-p)^{2}+1.75 p(1-p)^{3} \\
& +9.97 p^{3}(1-p) \\
B= & 2.83 p(1-p)(1-2 p)+(1-p)^{4} \\
C= & 2 p^{2}(1-p)^{2}
\end{aligned}
$$

and

$$
D=p^{4} .
$$

Once we get $\bar{\epsilon}(p)$ for $p>0.5$ from Eq. $(5), \bar{\epsilon}(1-p)$ can be obtained by using Keller's theorem.

In Fig. 3 the values of $\bar{\epsilon}$ for the case $\epsilon_{1}=1$, $\epsilon_{2}=10^{-10}$ is plotted as a solid line. Compared with the effective-medium theory ${ }^{7}$ (solid-circle line), the most striking feature of the new result is the existence of two sharp variations in $\bar{\epsilon}$, the percolation thresholds, as opposed to the one threshold at $p=0.5$ seen in the simple effective-medium case. The value of the upper percolation threshold, ${ }^{8} p_{c}^{u}=0.551$, can be obtained from Eq. (5) by first letting $\left|\epsilon_{2}\right| \rightarrow 0$ and then setting $\left|\bar{\epsilon} / \epsilon_{1}\right| \rightarrow 0$. Mathematically, the necessity of having a second percolation threshold at $p_{c}^{L}=0.449$ follows directly from Keller's theorem and the fact that $p_{c}^{u} \neq 0.5$. Physically, the two percolation thresholds are related to the formation of two types of connected networks. At $p=p_{c}^{L}$, an infinite network of $\epsilon_{1}$ materials is formed in which two $\epsilon_{1}$ squares are classified as being connected if they have either a common side or a common corner. The sharp rise in the effective conductivity in this case is ascribable to the leakage of the conducting current around 


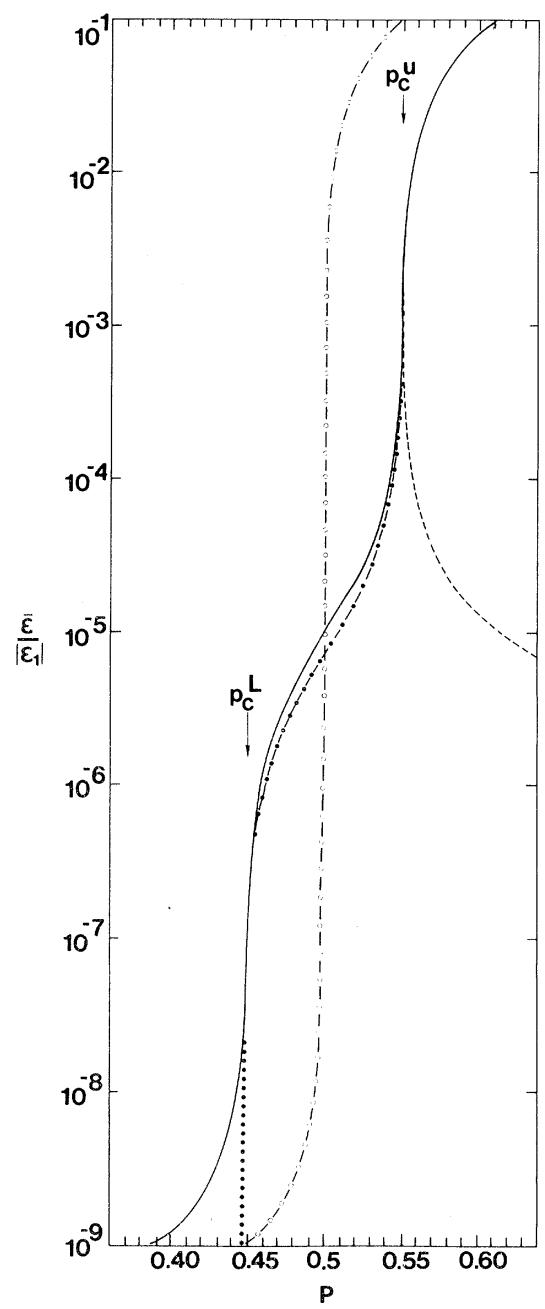

FIG. 3. Normalized effective dielectric constant as a function of $p$. Solid line denotes the case for $\epsilon_{1}=1$, $\epsilon_{2}=10^{-10}$. Dashed line and dotted line denote the real and imaginary parts of $\bar{\epsilon}$, respectively, for the case $\epsilon_{1}=i$, $\epsilon_{2}=10^{-10}$. Dotted-dashed line delineates the section over which the curves for the real and imaginary parts are coincident on the scale of the figure. Circle-dashed line denotes Bruggeman's effective medium theory with $\epsilon_{1}=1, \epsilon_{2}=10^{-10}$.

the connecting corners, since these must be the conductivity bottleneck of the network. On the other hand, at $p=p_{c}^{u}$ an infinite network of $\epsilon_{1}$ squares is formed in which every $\epsilon_{1}$ square must share at least one common side with another $\epsilon_{1}$ square. It is clear that another jump in the effective conductivity must ensue when this happens. An interesting case arises when $\left|\epsilon_{1} / \epsilon_{2}\right| \rightarrow \infty$. In that limit, only one of the percolation thresholds is observable. However, depending on whether it is $\left|\epsilon_{1}\right| \rightarrow \infty$ (normal conductor-superconductor composite) or $\left|\epsilon_{2}\right| \rightarrow 0$ (normal metal-dielectric mix- ture), the observed threshold can be either $p_{c}^{L}$ or $p_{c}^{u}$, respectively. To further elaborate on this point, we note that for $\left|\epsilon_{2}\right|=0$, our system is equivalent to a site percolation model with nearest-neighbor bonds. However, if $\left|\epsilon_{1}\right| \rightarrow \infty$, then the system reduces to a different site percolation model with both the nearest-neighbor and the next-nearestneighbor bonds. This nonuniqueness in the limiting behavior as $\left|\epsilon_{1} / \epsilon_{2}\right| \rightarrow \infty$ is a direct consequence of the variable nature of the third dielectric scale, $\sqrt{\epsilon_{1} \epsilon_{2}}$.

For complex dielectric constants, $\epsilon_{1}=\left|\epsilon_{1}\right| \exp \left(i \phi_{1}\right)$ and $\epsilon_{2}=\left|\epsilon_{2}\right| \exp \left(i \phi_{2}\right)$, analysis shows that the magnitude of $\bar{\epsilon}$ still follows the similar behavior as shown by the solid line. The phase $\phi$, on the other hand, has three roughly constant regions with sudden jumps at the two threshold: $\phi \cong \phi_{2}$ for $0 \leq p<p_{c}^{L}, \phi=\left(\phi_{1}+\phi_{2}\right) / 2$ for $p_{c}^{L}<p<p_{c}^{u}$, and $\phi \cong \phi_{1}$ for $p_{c}^{u}<p \leq 1$. As an example, we let $\epsilon_{1}$ be purely imaginary (conductor) and $\epsilon_{2}$ be purely real (dielectric), and $\left|\epsilon_{2} / \epsilon_{1}\right|=10^{-10}$. The real and imaginary parts of $\bar{\epsilon}$ are plotted in Fig. 3. It is seen that whereas the real part exhibits a sharp peak at $p_{c}^{u}$, it is monotone in $p$ at $p_{c}^{L}$. In view of the work by Bergman et al. ${ }^{9}$ in which they concluded that the real part of the dielectric constant diverges from both sides of the percolation threshold, the present result shows that only the behavior at $p_{c}^{u}$ conforms to that prediction. Near $p_{c}^{u}, \bar{\epsilon}$ can be written as

$$
\bar{\epsilon}=\left\{\begin{array}{l}
1.8 \epsilon_{1}\left(p-p_{c}^{u}\right)+\frac{0.1 \sqrt{\epsilon_{1} \epsilon_{2}}}{\left(p-p_{c}^{u}\right)}, p>p_{c}^{u} \\
\sqrt{\epsilon_{1} \epsilon_{2}}\left[\frac{0.1}{\left(p_{c}^{u}-p\right)}-1\right], p<p_{c}^{u}
\end{array}\right.
$$

where it is assumed that $\left|\epsilon_{2} / \epsilon_{1}\right| \rightarrow 0$, and $1 \gg\left|p-p_{c}^{u}\right| \gtrsim\left|\epsilon_{2} / \epsilon_{1}\right|$. At $p=p_{c}^{L}$, all divergencies or approaches to zero take the form $\left|p-p_{c}^{L}\right|^{ \pm 1}$.

Three implications of this work should be mentioned:

(i) From the physical reasoning that the two thresholds arise from two different types of connected networks, it can be inferred that a twodimensional hexagonal lattice must have one threshold at $p=0.5$, whereas a triangular lattice is predicted to have two thresholds.

(ii) In a three-dimensional simple cubic lattice composed of good-conductor and poor-conductor cubes, three conductivity thresholds are possible since two cubes can be connected by either a common corner, edge, or face. 
(iii) In a three-dimensional composite in which the constituents have irregular geometric shapes, the conductivity transtion might be smeared due to the following scenario. If the volume fraction of the good conductor $p$ is increased from zero, sharp protrusions of the conducting constituents would establish contacts first, forming a connected network. As $p$ is increased further, there can be a continuous series of thresholds caused by increasingly more effective geometric contacts between conducting grains in the connected network, each causing some increase in the effective conductivity. This geometric mechanism for broadening the conductivity transition offers an alternative to quantum-mechanical tunneling as the explanation for the smeared transition seen in sputtered granular-metal films. ${ }^{10}$

We wish to thank R. W. Cohen for many interesting discussions.
${ }^{*}$ Present address: Courant Institute of Mathematical Science, New York University, 251 Mercer Street, New York, New York 10012.

${ }^{1}$ See, for example, S. Kirkpatrick, Rev. Mod. Phys. 45, 574 (1973); many articles on percolations can also be found in Electrical Transport and Optical Properties of Inhomogeneous Media, Proceedings of the First Conference on the Electrical Transport and Optical Properties of Inhomogeneous Media, edited by J. C. Garland and D. B. Tanner (AIP, New York, 1978); J. W. Essam, Rep. Prog. Phys. 43, 833 (1980); H. Kesten, Commun. Math. Phys. 74, 41 (1980); also see P. M. Kogut and J. P. Straley, J. Phys. C 12, 1 (1979); 12,2151 (1979). The multiple critical points in Kogut and Straley's paper are induced by the presence of more than two physical components in the model. In our case, the third conductivity scale arising from the corner-corner interaction plays the role of the third physical component. However, unlike a truly independent third component, the corner conductance does not have a value independent from the other two.

${ }^{2}$ R. Landauer, in Electrical Transport and Optical Properties of Inhomogeneous Media, Proceedings of the First Conference of the Electrical Transport and Opti- cal Properties of Inhomogeneous Media, edited by J. C. Garland and D. B. Tanner (AIP, New York, 1978), p. 2.

3P. Sheng, Phys. Rev. Lett. 45, 60 (1980).

${ }^{4}$ M. F. Sykes and J. W. Essam, Phys. Rev. 133, 1310 (1964).

${ }^{5}$ R. Landauer, J. Appl. Phys. 23, 779 (1952).

6J. B. Keller, J. Math. Phys. $\underline{5}, 548$ (1964). The theorem has been generalized by K. S. Mendelson, J. Appl. Phys. 46, 917 (1975).

${ }^{7}$ D. A. G. Bruggeman, Ann. Phys. (Leipzig) 24, 636 (1935).

${ }^{8}$ This number should be compared with the threshold of site percolation on a simple square lattice, $p=0.59$. See B. P. Watson and P. L. Leath, Phys. Rev. B 9 , 4893 (1974); also P. Reynolds et al., ibid. 21, 1223 (1980).

${ }^{9}$ D. J. Bergman and Y. Imry, Phys. Rev. Lett. 39, 1222 (1977).

${ }^{10}$ B. Abeles, Appl. Solid State Sci. $\underline{6}, 1$ (1976). Annealing of the granular-metal films, which tends to make conducting grains more spherical, is known to sharpen the percolation transition. This fact is consistent with our view of the broadening mechanism. 